\title{
Methodological approaches to risk assessment of real investment projects
}

\author{
Ekaterina Bogomolova ${ }^{1, *}$ \\ ${ }^{1}$ State University of Management, Ryazanskiy prospect, 99, Moscow, 109542, Russia
}

\begin{abstract}
Risk management is impossible without systematically assessing the level of significance of the risks identified in the project. Within the scope of the paper, the distinctive features of various qualitative and quantitative methods for assessing the risks of real investment projects are emphasized. The paper analyzes some of the existing methods of risk assessment in terms of their advantages and disadvantages of application in order to facilitate the selection of the most convenient method for a particular project.
\end{abstract}

\section{Introduction}

Nowadays, there is a tendency of growth of investment activity in the real sector of the economy. When implementing investment projects, there is a likelihood of unforeseen situations that often lead to a decrease in project revenues or to its complete elimination. Consequently, the real investment projects are implemented in conditions of constant occurrence of various types of risks.

Risk analysis is a systematic process of assessing the level of significance of risks identified in a project. One of the fundamental stages of the risk management cycle is the risk analysis of innovation activities. Traditionally, the process of analyzing and assessing risks includes the following steps: identifying the causes and sources of risk, identifying all possible risks that may be inherent in the project under consideration, analyzing the degree of impact of individual types of risk, calculating and justifying the economic feasibility of a project, calculating the acceptable level of risk, developing and conducting measures to minimize the identified risks of a project. Not only the efficiency of risk management but also the result of the investment project, investment activity and competitiveness of the enterprise as a whole depend on how well the risk analysis will be conducted.

\section{Materials and Methods}

The results of research by a number of Russian and foreign scientists [1] indicate that each of the currently existing methods of risk analysis has its own scope and procedures. However, with all the variety of methods for assessing the level of risk, there is the problem of choosing the most acceptable one. Proper assessment of the strengths and weaknesses of the risk analysis methods allows the project team to quickly choose the correct method,

${ }^{*}$ Corresponding author: ea_bogomolova@guu.ru 
which ultimately leads to an improvement in the risk management process in a project. Successful risk management requires a risk analysis process based on a scientific approach supported by qualitative and quantitative methods [2].

The purpose of a qualitative analysis is to determine the factors, scope and types of risk. Qualitative analysis involves the classification and identification of risk, the determination of risk occurrence factors, the determination of possible damage, and the development of measures to minimize negative consequences. Qualitative analysis of risk includes an analysis of the likelihood of risks, an assessment of their impact on the objectives of a project, an analysis of the causes of risk, an analysis of the significance of risk, and the compilation of a list of priority risks. Various tools are used to collect and analyze data, such as: SWOT (Strengths, Weaknesses, Opportunities, Threats) and PEST analysis, the Ishikawa causal diagram. Each of these methods is widely used by Western and domestic analysts [3].

SWOT analysis is one of the most common methods of risk analysis. It is based on a cross-sectional analysis, which takes into account and compares both internal and external factors affecting the project. The advantage of the SWOT analysis is simplicity. However, the method also has disadvantages. For example, the results of using the SWOT analysis will depend entirely on the completeness and quality of the initial information. There is also a high probability of making mechanical errors that are sometimes difficult to detect.

PEST analysis (Political, Economic, Social, Technological) is a simple and widely used tool that allows analyzing the political, economic, socio-cultural, and technological factors that have an impact on a project. PEST analysis is often used in conjunction with the aforementioned SWOT analysis - these two tools complement each other. PEST analysis is a mechanism that can reduce the impact of potential threats; it is also quite easy to use. However, when conducting a PEST analysis, it should be remembered that environmental factors are very dynamic. Changes in the external environment that may affect the project are often unidentifiable at the initial stage. Also carrying out a PEST analysis takes a lot of time; it is very expensive method, since there are difficulties in obtaining the necessary amount of information about the external environment. The lack of available and relevant information can lead to errors in the analysis. Thus, in order to get a realistic picture of the external environment, PEST analysis should be used with other tools, such as SWOT analysis [4].

\section{Results}

Graphic methods are widely used in identification and assessment of risks. The most popular causal diagram proposed by Kaoru Ishikawa, one of the greatest Japanese management theorists. In various sources, the diagram is referred to as a "fishbone" or "problem tree". The Ishikawa diagram allows analyzing and structuring all risk factors that influence the result of the analysis. The tool is very practical for analyzing the causes leading to the occurrence of risks [5]. The diagram is convenient and easy to use. It allows us to graphically display the relationship between risk and the reasons for which the risk occurs. However, this method is not applied separately from other risk analysis tools. It is most convenient to apply the Ishikawa diagram in the initial analysis as a part of a comprehensive risk assessment. The results of the analysis carried out using the Ishikawa diagram, as a rule, are subjective, since it is rather difficult to correctly determine the relationship between the problem under investigation and its causes, especially if the problem is complex.

Based on the information obtained in the course of qualitative analysis, it becomes possible to carry out a quantitative analysis. Quantitative analysis of risks is based on information obtained in the course of qualitative analysis and involves the numerical 
determination of individual risks and the risk of a project as a whole. The main actions at this stage are to determine the numerical values of the occurrence of risk events and their consequences, to conduct a quantitative assessment of the degree of risk, to determine the acceptable level of risk in the current situation. Nowadays, there are many quantitative risk assessment methodologies. The most widely used methods for quantitative analysis of project risks are: statistical methods of assessment, expert assessment method, analogy method, group of analytical methods. Let us briefly consider each of the mentioned methods [6].

Statistical methods of risk assessment imply the determination of the probability of occurrence of a risk event, based on the available data of the previous period. Statistical methods make it possible to take into account and analyze various scenarios, to consider different factors within the framework of one approach. Currently, statistical methods for quantitative risk assessment are considered to be one of the most common methods. They have certain advantages, for example, the simplicity of mathematical calculations. The disadvantages include the need for a large number of observations and arrays of data. Also a significant disadvantage is the need to use probabilistic characteristics.

The essence of the expert assessment method is to attract people who have experience with a similar project in the not so distant past. Expert assessment is used to identify potential costs, assess the likelihood of risk events, interpret available data, and identify tools that can be used to minimize risks. In the field of risk management, there are many methods developed for conducting expert analysis, as well as their computer support, which makes expert analysis a widely used method of risk analysis. However, the method has disadvantages, the main of which is the difficulty of engaging independent experts and, as a result, the subjectivity of the obtained estimates [7].

The Delphi method is a variety of expert assessment methods, but it is a bit different from them. The procedure of analyzing by the Delphi method is much more formalized and rigorous. The basic concept is to obtain consensus by using a group of experts to come to a convergent solution for a specific problem. This is particularly useful in developing estimates of probability for future events where the impact of risk is large and critical. This method allows reducing the subjectivity of assessments, which is undoubtedly a plus. However, this method of analysis requires a lot of time and great organizational efforts. The disadvantages of the Delphi method are manifested through the limitations of its application for technical risks and dependence on the actual competence of specialists. In addition, the method may take longer than expected, depending on the iteration of input of experts. The method is especially convenient when assessing potential risk events in new types of activities in which the impact of risk is of key importance [8].

To calculate the probabilities of losses, the method of analogies is often used, which consists in analyzing all the available data related to the implementation of similar projects in the past. The use of this method is associated with some difficulties. For example, each project is somehow unique and has its own specific features of a different nature, so the selection of a project-analogue is sometimes impossible. It is also extremely difficult to assess how the level of risk of a project-analogue corresponds with the project in question. Moreover, an analysis of the available literature made it possible to identify a significant gap related to the methodological developments in the procedure for conducting risk analysis using the method of analogies.

The whole array of analytical methods can be divided into two subgroups depending on the attraction of probability distributions: methods that don't take into account probability distributions; methods that take into account probability distributions. Methods without probability distributions are relatively "traditional" ways of considering risk and include: sensitivity analysis of project performance criteria; scenario analysis. 
Of all the methods presented, sensitivity analysis of the performance criteria is the simplest and, therefore, the most widely used method for quantitative risk assessment. Sensitivity analysis was created to show a change in a certain performance criterion (for example, NPV (Net Present Value)) when the value of a selected impact factor changes. Sensitivity analysis is based on identifying critical parameters of a project. It is advisable to use the sensitivity analysis in two cases: when determining factors most influencing the project; when calculating the influence of hardly predictable factors on the project's efficiency (used in the comparative analysis of projects). The essence is the simultaneous and consistent change of all verifiable project variables. This method eliminates the main disadvantage of a sensitivity analysis, and the deviations of the parameters are calculated taking into account their correlation. The relative importance of each proven variable is easily visible. The visibility of the maximum or minimum value of the critical parameter justifies financial investments and ultimately indicates how effective or inefficient the project is [8]. The method is quite simple in calculations, objective and theoretically transparent. Nevertheless, the disadvantages include: single factor method, underestimation of the correlation of individual factors. Also, the sensitivity diagram does not contain information about the possible probability of occurrence of the risk under consideration. Thus, when assessing by the sensitivity analysis method, it is necessary to single out factors independent of each other. If the factors are closely interrelated, it is more logical to choose another analysis technique, for example, the scenario analysis method, which is a development of the sensitivity analysis methodology.

Traditionally, three scenarios are built: pessimistic, optimistic, and most probabilistic. The problem of this method may be the need for a large amount of preparatory work for the selection and processing of information, which is mandatory for the proper building of scenarios. Insufficient information leads to blurring of the boundaries of scenarios and a decrease in their predictive value. There is also a limit on the number of possible variables to be analyzed. Otherwise, it is possible to obtain an excessively large amount of information. Thus, when using the method, it is recommended to carefully select information and meticulously choose experts. In essence, the method of scenarios is one of the methods of network diagrams that are most appropriate to use in large-scale projects.

Methods for assessing project risks with regard to probability distributions represent the second group of analytical methods. It is assumed that the building and calculations by the model are carried out in accordance with the principles of the probability theory. Nowadays, the most popular methods within this theory are: methods based on the use of decision theory ("decision tree"), Monte Carlo method.

The "decision tree" method helps to form a balanced picture of risks and benefits. A decision tree should be used when analyzing the risks of projects that have a foreseeable number of development options. The advantage of this type of analysis is that it makes it possible to assess the probability of each outcome. The method is quite simple in understanding and interpretation, reliable, allows working with a large amount of information without preliminary preparation procedures, so it can be applied to many different uncertain situations. The disadvantages include instability, since the reliability depends on obtaining accurate data at the very beginning. Even a small change in the input data can cause big changes in a tree. The decisions contained in a tree are based on expectations, and irrational expectations can lead to errors in a decision tree. Analysis of a decision tree can be useful in calculating the expected value of a project, in identifying alternative solutions for a project. In addition, analysis of a decision tree is useful in situations that require discrete estimates. In more complex situations, it is recommended to use simulation modeling, such as Monte Carlo or others. 


\section{Discussions}

Monte Carlo method - simulation using random numbers, is an effective way to analyze risks. The method is based on the building of a mathematical model for a project, taking into account the probability distributions of the project parameters. Monte Carlo method is the most difficult, but also the most correct method of accounting and assessing risks, since it most fully describes the whole range of uncertainties that the project may face. It makes sense to use the method when the predicted situation has a high degree of complexity and uncertainty. Monte Carlo simulation is a valuable method for analyzing risks, in particular those related to costs and expenses. However, when using the method, it is necessary to take care when determining the correlation and the values of the range of project variables. The disadvantages include the complexity of the simulated graphs and the need for specialized software. Therefore, it is advisable to work with abbreviated models, such as a project schedule or cost estimate. It should be noted that in the case of analyzing a specific risk, there is no need to use complex analysis methods that require computer support. Nevertheless, if we consider the entire project as a whole, then computer simulation is indispensable.

It should be noted that some projects do not require a quantitative analysis of risks. For example, in smaller projects, a qualitative analysis provides enough information to make appropriate decisions regarding risk minimization. However, risk analysis cannot be called complete only by quantitative methods, since a partial risk analysis using qualitative methods assesses risks separately, but does not reflect the overall project risk in full. Therefore, in most cases, it is recommended to use a qualitative and quantitative assessment in the complex.

\section{Conclusion}

All of the above methods are an important part of a toolkit for carrying out a comprehensive analysis of project risks. Understanding the strengths and weaknesses of risk assessment methods helps to make the right decision and choose the method that most closely corresponds to the requirements of a particular project. In current practice, it is necessary to use the whole range of different risk assessment methods. The use of many different types of analysis in the complex allows correctly assessing the existing risks and minimizing potential losses, thereby increasing the efficiency of the real investment project being implemented.

\section{References}

1. A. Larionov, MATEC Web of Conferences 106, 09022 (2017)

2. D. Kaliampakos, A. Benardos, A. Mavrikos, Tunnelling and Underground Space Technology 55, 236-244 (2016)

3. J. Richardson, M. Johnson, Algal Research 10, 16-24 (2015)

4. G. Lytvynchenko, Procedia - Social and Behavioral Sciences 230, 198-203 (2016)

5. Y. Panibratov, A. Larionov, Applied Mechanics and Materials 725-726, 1007-1012 (2015) doi:10.4028/www.scientific.net/AMM.725-726.1007

6. P.G. Grabovy, A.K. Orlov, Procedia Engineering 153, 195-202 (2016)

7. I. Lukmanova, M. Mishlanova, Bulletin of Irkutsk State Technical University 11, 294299 (2014)

8. S. Domnina, E. Savoskina, N. Shekhova, Procedia Engineering 153, 741-746 (2016) 